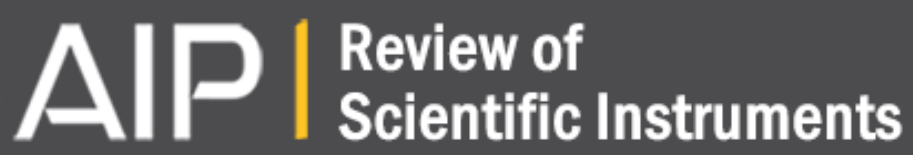

\section{Measuring coalignment of retroreflectors with large lateral incoming-outgoing beam offset}

Daniel Schütze, David Farrant, Daniel A. Shaddock, Benjamin S. Sheard, Gerhard Heinzel, and Karsten Danzmann

Citation: Review of Scientific Instruments 85, 035103 (2014); doi: 10.1063/1.4866682

View online: http://dx.doi.org/10.1063/1.4866682

View Table of Contents: http://scitation.aip.org/content/aip/journal/rsi/85/3?ver=pdfcov

Published by the AIP Publishing

\section{Articles you may be interested in}

Faraday-effect polarimeter-interferometer system for current density measurement on EASTa)

Rev. Sci. Instrum. 85, 11D405 (2014); 10.1063/1.4889777

2D image of local density and magnetic fluctuations from line-integrated interferometry-polarimetry measurementsa)

Rev. Sci. Instrum. 85, 11D403 (2014); 10.1063/1.4884788

Portable long trace profiler: Concept and solution

Rev. Sci. Instrum. 72, 3198 (2001); 10.1063/1.1384452

Energetic quantum limit in large-scale interferometers

AIP Conf. Proc. 523, 180 (2000); 10.1063/1.1291855

Integrated scatter plate and projection lens for scatter plate interferometer

Rev. Sci. Instrum. 69, 1587 (1998); 10.1063/1.1148813

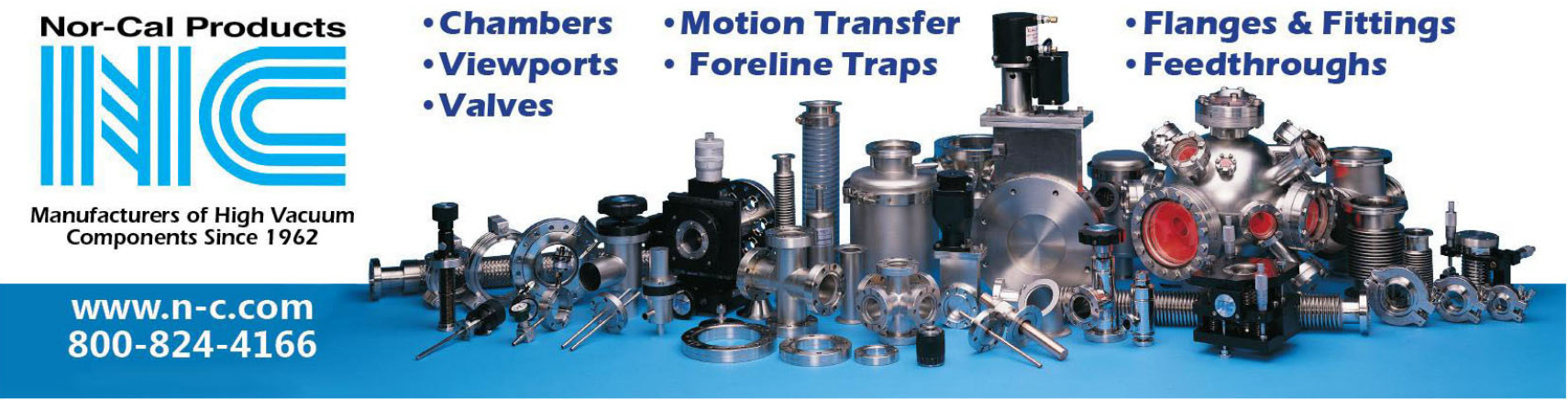




\title{
Measuring coalignment of retroreflectors with large lateral incoming-outgoing beam offset
}

\author{
Daniel Schütze, ${ }^{1, a)}$ David Farrant, ${ }^{2}$ Daniel A. Shaddock, ${ }^{3}$ Benjamin S. Sheard, ${ }^{1}$ \\ Gerhard Heinzel, ${ }^{1}$ and Karsten Danzmann ${ }^{1}$ \\ ${ }^{1}$ Max Planck Institute for Gravitational Physics (Albert Einstein Institute) and Institute for Gravitational \\ Physics, Leibniz Universität Hannover, Callinstr. 38, 30167 Hanover, Germany \\ ${ }^{2}$ Commonwealth Scientific and Industrial Research Organisation, Bradfield Road, Lindfield, \\ NSW 2070, Australia \\ ${ }^{3}$ Centre for Gravitational Physics, Australian National University, Acton, ACT 0200, Australia
}

(Received 6 January 2014; accepted 11 February 2014; published online 4 March 2014)

\begin{abstract}
A method based on phase-shifting Fizeau interferometry is presented with which retroreflectors with large incoming-outgoing beam separations can be tested. The method relies on a flat Reference Bar that is used to align two auxiliary mirrors parallel to each other to extend the aperture of the interferometer. The method is applied to measure the beam coalignment of a prototype Triple Mirror Assembly of the GRACE Follow-On Laser Ranging Interferometer, a future satellite-to-satellite tracking device for Earth gravimetry. The Triple Mirror Assembly features a lateral beam offset of incoming and outgoing beam of $600 \mathrm{~mm}$, whereas the acceptance angle for the incoming beam is only about $\pm 2 \mathrm{mrad}$. With the developed method, the beam coalignment of the prototype Triple Mirror Assembly was measured to be $9 \mu \mathrm{rad}$ with a repeatability of below $1 \mu \mathrm{rad}$. () 2014 AIP Publishing LLC. [http://dx.doi.org/10.1063/1.4866682]
\end{abstract}

\section{INTRODUCTION}

Retroreflectors are optical devices with applications ranging from everyday items such as cat's eye reflectors to upto-date science experiments. ${ }^{1-5}$ Their key parameter is how well they retroreflect an incoming beam, in other words: How well are incoming and outgoing beam coaligned? This property has been studied thoroughly over the years. While in the early days, dot patterns retrieved from a pinhole and an autocollimator were studied, ${ }^{6,7}$ the techniques have been refined by the use of interferometric methods such as phase-shifting Twyman-Green or Fizeau interferometers. ${ }^{8-15}$ The interferograms are recorded by fully illuminating the retroreflector under test, which is usually a corner cube.

A challenge arises when the retroreflector has a large aperture. The straightforward approach of just scaling the interferometer aperture can be a challenging and unreasonably expensive task. One possible solution for applications involving large aperture optics is subaperture stitching interferometry. ${ }^{16}$ Yet this is not possible for retroreflectors, since incoming and outgoing beams are laterally displaced by twice the distance from the center of the retroreflector. Other widely used methods are "Ritchey-Common" or "Skip-Flat" tests. ${ }^{17-19}$ However, these methods require large angles of incidence, which might not be supported by the specific retroreflector.

A current example of such a retroreflector is the Triple Mirror Assembly (TMA) which will be used in GRACE Follow-On, a satellite-to-satellite tracking Earth gravity field recovery mission featuring the first inter-satellite Laser Ranging Interferometer. ${ }^{20}$ The TMA resembles the Lateral Transfer Hollow Retroreflector ${ }^{\mathrm{TM}}$ fabricated by PLX Inc., ${ }^{21-23}$ yet

a)Electronic mail: Daniel.Schuetze@ aei.mpg.de special effort has to be undertaken to meet the specific requirements of the GRACE Follow-On satellite mission. A prototype TMA has been designed by a consortium led by the Australian National University (ANU) and fabricated and measured by the Commonwealth Scientific and Industrial Research Organisation (CSIRO) ${ }^{24}$ see Fig. 1. Three gold-coated substrates serve as mirrors TMA1, TMA2, TMA3, which are mounted perpendicular with respect to each other. A CarbonFiber-Reinforced Polymer (CFRP) tube is used as rigid structure to install mirror TMA1 with a $600 \mathrm{~mm}$ lateral offset to mirrors TMA2, TMA3. Virtually, this device mimics a corner cube with reflecting surfaces only where the incoming beam impinges. This limits the incidence angle to about \pm 2 mrad.

We present a method based on phase-shifting Fizeau interferometry to measure beam coalignment of large retroreflectors with lateral incoming-outgoing beam offsets up to $600 \mathrm{~mm}$ (in principle extendable). We use this method to measure the beam coalignment of the GRACE Follow-On prototype TMA.

\section{SETUP}

We propose to measure beam coalignment for large retroreflectors such as the prototype TMA with the setup shown in Fig. 2. The key feature of this setup is a flat Zerodur $^{\circledR}$ Reference Bar (RB) which was fabricated at CSIRO. The RB is used to align the auxiliary mirrors M1, M2 parallel to each other. By this, the $150 \mathrm{~mm}$ diameter aperture of the homodyne phase-shifting interferometer (Wyko 6000) can be extended to the desired size. The RB surface properties were characterized using a "Skip-Flat" test: ${ }^{17-19}$ The RB flat surface has a rectangular shape of $650 \times 79 \mathrm{~mm}^{2}$ and was installed under a $40^{\circ}$ incidence angle leading to an effective aperture of $116 \times 79 \mathrm{~mm}^{2}$ which was slightly overfilled by the 


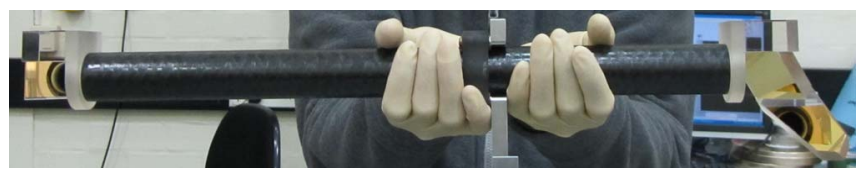

FIG. 1. Triple Mirror Assembly (TMA) designed by a consortium led by the Australian National University (ANU) and fabricated by the Commonwealth Scientific and Industrial Research Organisation (CSIRO). The mirrors TMA1 (left end of the tube) and TMA2, TMA3 (right end of the tube) are separated by $600 \mathrm{~mm}$ and aligned perpendicular to each other. The TMA is mounted by its bracket that is attached to the tube close to the center of mass.

$150 \mathrm{~mm}$ diameter aperture of the interferometer. The topography of the return flat had been previously measured (peak-tovalley $<70 \mathrm{~nm}$ ) and could be substracted. The results of the "Skip-Flat" test are shown in Fig. 3. The RB shows a concave topography with a peak-to-valley of about $600 \mathrm{~nm}$.

Let us now follow the beam path in Fig. 2. The beam coming from the homodyne phase-shifting interferometer (Wyko 6000) is split into two parts using mirror M1. One part traverses the TMA counterclockwise, the other, reflected by a second mirror M2, which is parallel to M1, travels clockwise through the TMA. The TMA is arranged in a way that interferograms of clockwise and counterclockwise propagating beams can be observed simultaneously on the CCD of the interferometer with interferograms of the reflections from both sides of the $R B\left(R B_{L}, R B_{R}\right)$.

There are two coordinate systems shown in Fig. 2, the unprimed interferometer-bound ICS and the primed TMA-bound TCS. The $z$-axis of the ICS points along the outgoing interferometer beam. The $x$-axis and $y$-axis of the ICS lie in the plane of the interferometer's CCD camera on which the interferograms are recorded. The $x$-axis denotes the horizontal direction, the $y$-axis the vertical direction. The TCS is obtained by rotating the ICS around the $z$-axis by the TMA tube angle $\phi_{\text {tube }}$. The $x^{\prime}$-axis of the TCS points from TMA mirrors TMA2, TMA3 to TMA1. During the measurements, the TMA tube angle $\phi_{\text {tube }}$ is either $180^{\circ}$ or $0^{\circ}$, with a slight deviation from these nominal values by only a few degrees for practical reasons.

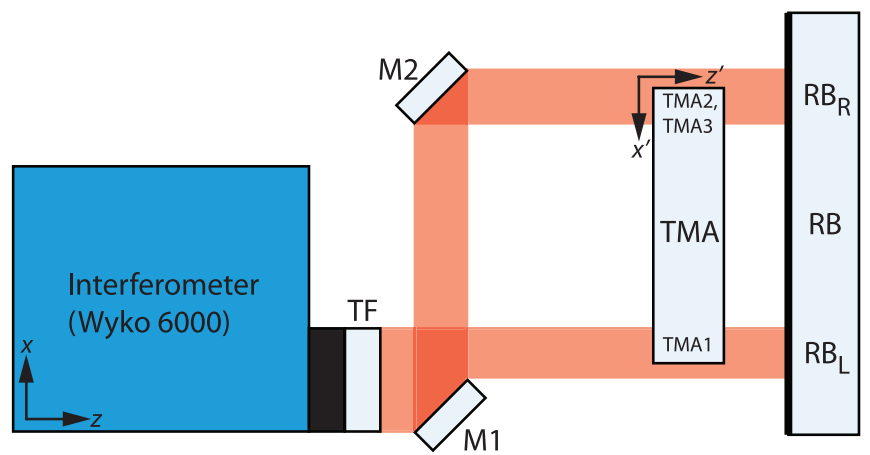

FIG. 2. Setup to measure beam coalignment of the Triple Mirror Assembly prototype (TMA) with a homodyne phase-shifting interferometer (Wyko 6000 ) and a flat Reference Bar (RB) to align the two mirrors M1, M2 parallel to each other. $\mathrm{RB}_{\mathrm{L}}$ and $\mathrm{RB}_{\mathrm{R}}$ denote the "left" and "right" side of the RB. TF is the Transmission Flat the reflection of which generates the reference beam for the interferometric measurement. TMA1-3 indicate the three mirrors of the TMA. The unprimed interferometer coordinate system (ICS) and the primed TMA coordinate system (TCS) are also shown.

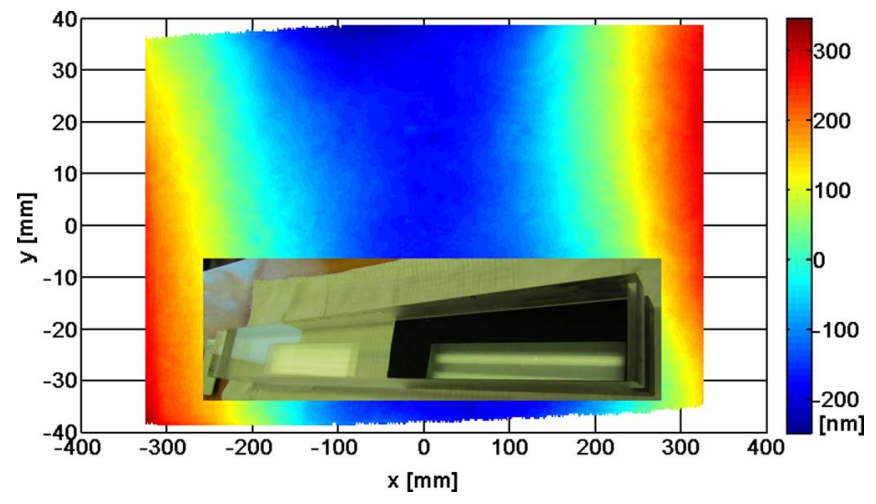

FIG. 3. Topography of the $650 \times 79 \mathrm{~mm}^{2}$ Reference Bar (RB) measured with the "Skip-Flat" test. The inset shows a photograph of the RB.

\section{DATA ANALYSIS}

We will now discuss how to obtain the TMA coalignment information from the interferograms recorded with the Wyko 6000 interferometer. In particular, we will explain how to remove the main error sources, which are imperfect parallelism between mirrors M1, M2 and non-flatness of the RB.

The Wyko 6000 interferometer performs a phase-shifting measurement by moving the Transmission Flat. From the measurement, a two-dimensional map in the $x$-y-plane with a phase value for each pixel of the interferometer CCD camera is obtained. With the spatial scale of the CCD pixels given by the manufacturer, $x_{\text {scale }}=0.2907\left[\mathrm{~mm} /\right.$ pixel], $y_{\text {scale }}$ $=0.33920[\mathrm{~mm} / \mathrm{pixel}]$, and the interferometer wavelength, $\lambda=632.8 \mathrm{~nm}$, the measurement can be converted into pathlength variations over an area. A typical measurement is shown in Fig. 4. In our case, each pathlength variations map consists of four distinct sectors $K_{\mathrm{i}}(x, y), i=\mathrm{RB}_{\mathrm{L}}$, $\mathrm{RB}_{\mathrm{R}}, \mathrm{TMA}_{\mathrm{R}}, \mathrm{TMA}_{\mathrm{L}}$, which are schematically drawn in the inset, lower left corner of Fig. 4. The four sectors result from beams reflected off the left and right side of the Reference Bar, $\mathrm{RB}_{\mathrm{L}}$ and $\mathrm{RB}_{\mathrm{R}}$, and from beams propagating clockwise and counterclockwise through the TMA, TMA $A_{R}$ and $\mathrm{TMA}_{\mathrm{L}}$.

The partial derivatives of each sector $\frac{\partial}{\partial x} K_{\mathrm{i}}(x, y)$ and $\frac{\partial}{\partial y} K_{\mathrm{i}}(x, y)$ can be interpreted as tilt angles of the return beam

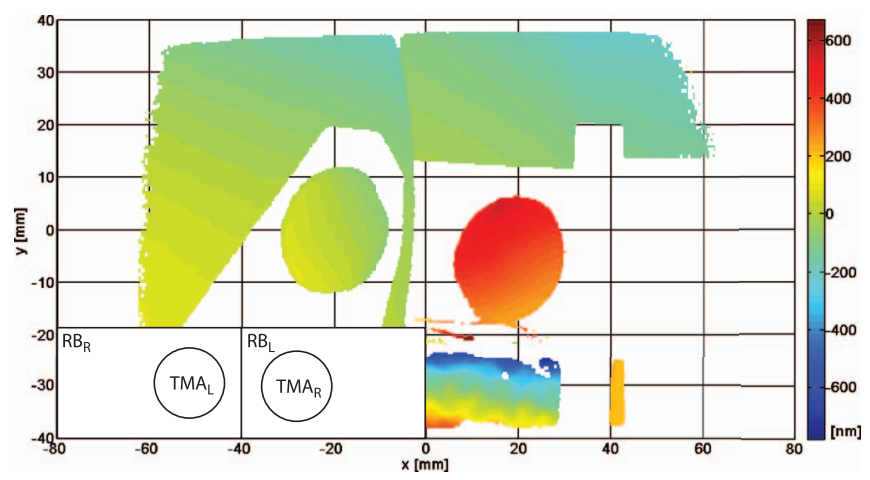

FIG. 4. Typical measurement of pathlength variations over the $x-y-p l a n e$. The measurement divides in four distinct sectors which are schematically drawn in the inset, lower left corner: $\mathrm{RB}_{\mathrm{L}}, \mathrm{RB}_{\mathrm{R}}$ result from beams reflected off the left and right side of the Reference Bar, $\mathrm{TMA}_{R}, \mathrm{TMA}_{\mathrm{L}}$ from beams propagating clockwise and counterclockwise through the TMA. 
with respect to the interferometer reference beam which is the beam reflected off the Transmission Flat (TF, see Fig. 2). The partial derivative along the $y$-axis gives the angles

$$
\varphi_{\mathrm{x}, \mathrm{i}}=\arctan \left(\frac{\partial}{\partial y} K_{\mathrm{i}}(x, y)\right),
$$

with $i=\mathrm{RB}_{\mathrm{R}}, \mathrm{RB}_{\mathrm{L}}, \mathrm{TMA}_{\mathrm{L}}, \mathrm{TMA}_{\mathrm{R}}$. Those angles describe counterclockwise rotations around the $x$-axis. Analogously, the angles

$$
\varphi_{\mathrm{y}, \mathrm{i}}=-\arctan \left(\frac{\partial}{\partial x} K_{\mathrm{i}}(x, y)\right),
$$

$i=\mathrm{RB}_{\mathrm{R}}, \mathrm{RB}_{\mathrm{L}}, \mathrm{TMA}_{\mathrm{L}}, \mathrm{TMA}_{\mathrm{R}}$, describe counterclockwise rotations around the $y$-axis.

The TMA beam coalignment error in ICS coordinates for a counterclockwise propagating beam is denoted by $\delta_{\mathrm{q}}$, $q=x, y$. Here, $\delta_{\mathrm{x}}$ corresponds to a counterclockwise rotation of the outgoing beam around the $x$-axis with respect to the incoming beam (vertical coalignment error), whereas $\delta_{\mathrm{y}}$ denotes a counterclockwise rotation of the outgoing beam around the $y$-axis (horizontal coalignment error). We can obtain $\delta_{\mathrm{q}}, q=x, y$, from

$$
\delta_{\mathrm{q}}=0.5 \cdot\left(\varphi_{\mathrm{q}, \mathrm{TMA}_{\mathrm{L}}}-\varphi_{\mathrm{q}, \mathrm{TMA}_{\mathrm{R}}}\right)-\varphi_{\mathrm{q}, \mathrm{M}},
$$

where $\varphi_{\mathrm{q}, \mathrm{M}}$ is the coalignment error of a counterclockwise propagating beam passing the two mirrors M2, M1 due to their non-perfect parallelism. We can calculate

$$
\varphi_{\mathrm{q}, \mathrm{M}}=0.5 \cdot\left(\varphi_{\mathrm{q}, \mathrm{RB}_{\mathrm{R}}}-\varphi_{\mathrm{q}, \mathrm{RB}_{\mathrm{L}}}\right)+\varphi_{\mathrm{q}, \mathrm{RBpolish}},
$$

$q=x, y$, with the polish angles $\varphi_{\mathrm{q}, \mathrm{RBpolish}}$ between the right and left side of the Reference Bar which are obtained from the previously performed "Skip-Flat" test, cf. Sec. II.

Both Eqs. (3) and (4) follow from simple geometrical considerations and have been verified by raytracing simulations. The simulations rely on small angle approximations for rotations $(\sin \phi \approx \phi, \cos \phi \approx 0)$ and treat the TMA as a perfect retroreflector with a small coalignment error which is independent of the incidence angle of the incoming beam. This simplification is valid, since for a TMA with nominal coalignment errors of up to $50 \mu \mathrm{rad}$ and incident beam angles of up to $\pm 2 \mathrm{mrad}$, this approximation is correct to $50 \mathrm{nrad}$, as our simulations have shown. Incident beam angles much higher than $\pm 2 \mathrm{mrad}$ can be excluded, since this is beyond the TMA clear opening angle. Furthermore, the expected nominal TMA coalignment error is about $10 \mu \mathrm{rad}$.

The root-mean-square of the TMA coalignment error

$$
\delta_{\mathrm{RMS}}=\sqrt{\delta_{\mathrm{x}}^{2}+\delta_{\mathrm{y}}^{2}}
$$

is coordinate independent. Yet to transform $\delta_{\mathrm{q}}, q=x, y$, from ICS to TCS coordinates, we need to know the angle $\phi_{\text {tube }}$ by which the TCS is rotated around the interferometer $z$-axis. Knowing the horizontal $(600 \mathrm{~mm})$ and vertical $(48 \mathrm{~mm})$ offset for a beam propagating through the TMA, $\phi_{\text {tube }}$ can be determined from the vertical offset of the $\mathrm{TMA}_{R}$ and $\mathrm{TMA}_{\mathrm{L}}$ sectors in the measurement (see Fig. 4). The alignment error of the ICS $z$-axis and TCS $z^{\prime}$-axis has only a small effect and can be neglected. The coalignment error $\delta_{\mathrm{q}^{\prime}, \mathrm{TMA}}, q^{\prime}=x^{\prime}, y^{\prime}$, in TCS coordinates is then obtained by a coordinate transformation of $\delta_{\mathrm{q}}$ from unprimed ICS to primed TCS coordinates.
The interferometric measurements are processed with an evaluation script written on Matlab ${ }^{\circledR}$. We have tested the script with a raytracing-based data simulator and could recover all simulation parameters within a few percent. During the evaluation process, the measurement sectors (see Fig. 4) are chosen by hand which leaves some dependence of the final result on the areas chosen for analysis. For this reason, each measurement was evaluated 10 times selecting areas manually each time. Also, the illuminated areas on the RB were estimated to calculate the angles $\varphi_{\mathrm{q}, \mathrm{RBp} o l i s h}, q=x, y$, from the RB "Skip-Flat" test results which were below $4 \mu \mathrm{rad}$ for all runs. All obtained parameters exhibited a standard deviation of less than $10 \%$.

\section{RESULTS}

The measurement was performed for different TMA orientations $\phi_{\text {tube }} \approx 180^{\circ}, 0^{\circ}$, mounting types, and compensation loads installed at the TMA bracket to study the effect of gravity which is expected to bend the tube and thus deform the mirror alignment. Since in the final application the TMA will operate in zero gravity, the mirrors were assembled in a way to show best performance under such conditions. Simulations have shown that, by mounting the TMA with a 2-point support close to the inserts (mirror-tube-interface), a center load of $30 \mathrm{~g}$ installed at the bracket, and a tubeangle $\phi_{\text {tube }}=180^{\circ}$ (we have used $\phi_{\text {tube }}=184^{\circ}$ for practical reasons), the tubebending effect of gravity can be compensated such that the test conditions come close to a zero-gravity environment.

The results are summarized in Table I. With the abovementioned nominal "zero-gravity" mounting, we achieve a root-mean-square coalignment error of $\delta_{\mathrm{RMS}}=9 \mu \mathrm{rad}$.

For center-mounting by the TMA bracket, the root-meansquare coalignment error is $\delta_{\mathrm{RMS}}=16 \mu \mathrm{rad}$ for $\phi_{\text {tube }}=186^{\circ}$ and $\delta_{\mathrm{RMS}}=41 \mu \mathrm{rad}$ for $\phi_{\text {tube }}=6^{\circ}$. The coalignment error is expected to be higher for center-mounting due to gravity causing TMA deformations. As one would expect intuitively, there is mainly an effect on the vertical coalignment error $\delta_{x^{\prime}, \text { TMA }}$, whereas the horizontal coalignment error $\delta_{y^{\prime}}$,TMA stays the same, since gravity causes the tube to bend around the $z^{\prime}$-axis.

TABLE I. TMA coalignment errors for different TMA orientations, mounting types, and center loads installed at the TMA bracket. "2-point" denotes a 2-point mounting of the TMA on small steel rods close to the TMA inserts (tube-glass-interface). The nominal "zero-gravity" mounting is a "2-point" mounting with a center load of $30 \mathrm{~g}$ and a tubeangle $\phi_{\text {tube }}=180^{\circ}$. "Bracket" stands for center-mounting the TMA by its bracket.

\begin{tabular}{lccccc}
\hline \hline Mounting & Load $(\mathrm{g})$ & $\phi_{\text {tube }}(\mathrm{deg})$ & $\begin{array}{c}\delta_{x^{\prime}, \mathrm{TMA}} \\
(\mu \mathrm{rad})\end{array}$ & $\begin{array}{c}\delta_{y^{\prime}, \mathrm{TMA}} \\
(\mu \mathrm{rad})\end{array}$ & $\begin{array}{c}\delta_{\mathrm{RMS}} \\
(\mu \mathrm{rad})\end{array}$ \\
\hline 2-point & 0 & 184 & -8 & 2 & 8 \\
& 30 & 184 & -9 & 3 & 9 \\
& 92 & 184 & -11 & 3 & 11 \\
& 183 & 184 & -14 & 2 & 14 \\
& 30 & 5 & -13 & 3 & 13 \\
\multirow{5}{*}{ Bracket } & 92 & 5 & -10 & 3 & 11 \\
& 0 & 186 & 16 & 5 & 16 \\
& 0 & 6 & -41 & 3 & 41 \\
\hline \hline
\end{tabular}


Compared to the "zero-gravity" mounting, the centermounting coalignment error $\delta_{x^{\prime}, \text { TMA }}$ for $\phi_{\text {tube }}=186^{\circ}$ differs by $25 \mu \mathrm{rad}$, whereas for $\phi_{\text {tube }}=6^{\circ}$, the difference is $-32 \mu \mathrm{rad}$. The opposite sign is the result of the tube bending in the opposite direction when turning it around the $z^{\prime}$-axis by $180^{\circ}$. The size of the effect only slightly differs which might be caused by TMA tube stiffness asymmetries.

For completeness, one has to mention that in a consecutive test with an Intellium $\mathrm{H} 2000$ interferometer and a different measurement method, the root-mean-square beam coalignment of the same TMA for "zero-gravity" mounting was measured to be $21 \mu \mathrm{rad}^{25}$ This discrepancy might be due to CFRP tube outgasing, CFRP-to-glass glue interface effects, or aging of the mirror bonds. The most likely candidate is deformation of the mirror mounting inserts due to glue shrinkage.

\section{v. CONCLUSION}

We have presented a novel phase-shifting Fizeau-type interferometry method for measuring beam coalignment of retroreflectors with large $(600 \mathrm{~mm}$, in principle extendable) lateral incoming-outgoing beam offset. The method relies on a flat Reference Bar to extend the interferometer aperture. Commercial Fizeau interferometers can be used and no dedicated large-aperture interferometer is required. We have investigated the beam coalignment of a GRACE Follow-On TMA prototype using the presented method and found its coalignment angle to be $9 \mu \mathrm{rad}$. Beam coalignments as small as $2 \mu \mathrm{rad}$ were measured with a repeatability of below $1 \mu \mathrm{rad}$.

\section{ACKNOWLEDGMENTS}

This work was partly funded by the "Bundesministerium für Bildung und Forschung" (BMBF, project number: 03F0654B), by the "Deutsche Forschungsgemeinschaft" (DFG) within the Cluster of Excellence QUEST (Centre for Quantum Engineering and Space-Time Research), and by the Australian Government's Australian Space Research Program (ASRP).
${ }^{1}$ P. L. Bender, D. G. Currie, R. H. Dicke, D. H. Eckhardt, J. E. Faller, W. M. Kaula, J. D. Mulholland, H. H. Plotkin, S. K. Poultney, E. C. Silverberg, D. T. Wilkinson, J. G. Williams, and C. O. Alley, Science 182, 229 (1973).

${ }^{2}$ C. F. Yoder, J. G. Williams, J. O. Dickey, B. E. Schutz, R. J. Eanes, and B. D. Tapley, Nature (London) 303, 757 (1983).

${ }^{3}$ M. Jeganathan and S. Dubovitsky, Proc. SPIE 4006, 838 (2000).

${ }^{4}$ A. Paolozzi, I. Ciufolini, and C. Vendittozzi, Acta Astronaut. 69, 127 (2011).

${ }^{5}$ G. Renzetti, New Astron. 23-24, 63 (2013).

${ }^{6}$ P. R. Yoder, J. Opt. Soc. Am. 48, 496 (1958).

${ }^{7}$ B. W. Joseph and R. J. Donohue, J. Opt. Soc. Am. 62, 727 (1972).

${ }^{8}$ A. Hilger Ltd., F. Twyman, and A. Green, "Improvements in finishing prisms or lenses or combinations of the same and in apparatus therefor," patent GB103832 (1917).

${ }^{9}$ Optical Shop Testing, edited by D. Malacara (Wiley, New York, 1978).

${ }^{10}$ J. Schwider, R. Burow, K. E. Elssner, J. Grzanna, R. Spolaczyk, and K. Merkel, Appl. Opt. 22, 3421 (1983).

${ }^{11}$ Y. Y. Cheng and J. C. Wyant, Appl. Opt. 24, 3049 (1985).

${ }^{12}$ D. A. Thomas and J. C. Wyant, J. Opt. Soc. Am. 67, 467 (1977).

${ }^{13}$ C. Y. Ai and K. L. Smith, Appl. Opt. 31, 519 (1992).

${ }^{14}$ M. S. Scholl, J. Opt. Soc. Am. A 12, 1589 (1995).

${ }^{15}$ J. Burke, K. L. Green, N. Raouf, J. A. Seckold, and B. F. Oreb, Proc. SPIE 7013, 701351 (2008).

${ }^{16}$ T. W. Stuhlinger, Proc. SPIE 0655, 350 (1986).

${ }^{17}$ J. B. Houston, Opt. Eng. 14, 88 (1975).

${ }^{18}$ T. S. Turner, Proc. SPIE 1752, 90 (1992).

${ }^{19}$ Z. Linchao, X. Bin, and X. Jingjiang, Proc. SPIE 7656, 76564W (2010).

${ }^{20}$ B. S. Sheard, G. Heinzel, K. Danzmann, D. A. Shaddock, W. M. Klipstein, and W. M. Folkner, J. Geod. 86, 1083 (2012).

${ }^{21}$ Z. Bleier and M. S. Lipkins, "Lateral transfer retroreflector and roof mirror for the same," U.S. patent 5,024,514 (1991).

${ }^{22}$ Z. Bleier, "Lateral transfer retroreflector assembly," U.S. patent 5,361,171 (1994).

23"Perfect optical alignment made simple," see http://www.plxinc.com/ documents/PERFECTOPTICALALIGNMENTMADESIMPLE11-bis.pdf.

${ }^{24}$ R. L. Ward, R. Fleddermann, M. Elliot, S. Francis, C. Mow-Lowry, D. Wuchenich, F. Gilles, M. Herding, K. Nicklaus, J. Brown, J. Burke, S. Dligatch, D. Farrant, K. Green, J. Seckold, M. Blundell, R. Brister, C. Smith, K. Danzmann, G. Heinzel, D. Schütze, B. S. Sheard, B. Klipstein, D. E. McClelland, and D. A. Shaddock, "The design and construction of a prototype lateral-transfer retro-reflector for inter-satellite laser ranging," Class. Quant. Grav. (submitted).

${ }^{25}$ R. Fleddermann, R. L. Ward, M. Elliot, D. Wuchenich, F. Gilles, M. Herding, K. Nicklaus, J. Brown, J. Burke, S. Dligatch, D. Farrant, K. Green, J. Seckold, M. Blundell, R. Brister, C. Smith, B. S. Sheard, G. Heinzel, K. Danzmann, B. Klipstein, D. E. McClelland, and D. A. Shaddock, "Testing the GRACE FO triple mirror assembly" (unpublished). 